\title{
Más allá de la terapia y la religión: una aproximación relacional a la construcción espiritual del bienestar
}

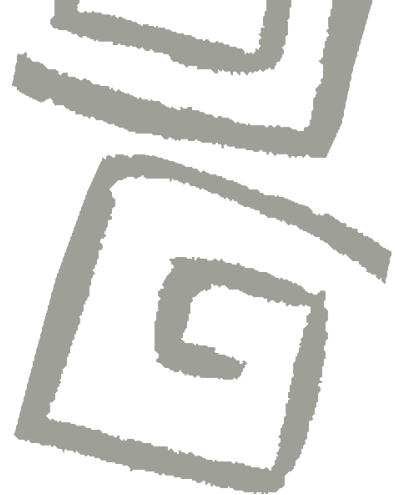

\author{
Beyond therapy and religion: a relational approach to \\ the spiritual construction of wellbeing
}

${ }^{1}$ Doctor en Antropología Social. Investigador Asistente, Consejo Nacional de Investigaciones Científicas y Técnicas, Ciudad Autónoma de Buenos Aires, Argentina. $\square$

\section{Nicolás Viotti ${ }^{1}$}

RESUMEN Teniendo en cuenta la discontinuidad entre lo corporal y lo anímico como una manifestación de la "gran división" moderna dominante, este trabajo se propone reflexionar críticamente sobre los alcances de esa división en el análisis de la dimensión terapéutica de la espiritualidad estilo Nueva Era. A partir de observación participante, entrevistas abiertas y convivencia con participantes del movimiento de espiritualidad de origen indio El Arte de Vivir, realizadas entre 2012 a 2017 en Buenos Aires, Argentina, se describen dos procesos. En primer lugar, el discurso institucional del movimiento y su localización en un régimen de disputas públicas en tensión entre lo terapéutico y lo religioso. En segundo lugar, analizamos un régimen de relaciones cotidiano que produce agenciamientos múltiples y una gramática espiritual. Mostramos entonces la importancia de atender una trama relacional y ensamblada que desafía el dualismo más convencional entre lo terapéutico/ religioso a la luz de un proceso de ordinarización más general de la gestión contemporánea del bienestar.

PALABRAS CLAVES Espiritualidad; Religión; Terapéutica; Argentina.

\begin{abstract}
Taking into account the discontinuity between the body and the soul as a manifestation of the dominant "great division" of modernity, this paper intends to reflect critically on the scope of this division in the analysis of the therapeutic dimension of New Age style spirituality. Through participant observation, open-ended interviews and shared experiences with members of a spiritual movement of Indian origin, The Art of Living, carried out between 2012 and 2017 in Buenos Aires, Argentina, we describe two processes. First, the institutional discourse of the movement and its location within public disputes regarding the tension between the therapeutic and the religious. Second, we analyze regimens of everyday relationships that produce multiple agencies and a spiritual grammar. We then suggest the importance of attending to a relational and assembled framework that challenges the more conventional dualism between the therapeutic/ religious in the light of a more general contemporary process of ordinarization in the management of well-being.
\end{abstract}

KEY WORDS Spirituality; Religion; Therapeutics; Argentina. 


\section{INTRODUCCIÓN}

El énfasis que ciertas prácticas identificadas modernamente como religiosas le dan a la construcción del bienestar no es algo novedoso. Esa articulación tiene una compleja historia en las sociedades occidentales, tanto en sus zonas subalternas, donde la distancia entre esos términos parece haber sido algo menos problemática, como en sus espacios más dominantes. Sea como fuere, es públicamente aceptado que los procesos de secularización han producido transformaciones significativas en esa relación al promover -con diferente grado de éxito y de intensidad- una diferenciación entre lo anímico, administrado por diferentes modalidades de lo religioso, y lo corporal, gestionado por los saberes y las prácticas médicas.

¿Qué es del orden de lo corporal y qué es del orden de lo espiritual? ¿Cómo se ha delimitado y estabilizado esa frontera? Nos interesa aquí llevar esas preguntas a un contorno específico: el de la denominada "espiritualidad contemporánea", que pone el acento en el trabajo intimista y relacional sobre el confort personal y que desafía las fronteras entre lo médico, lo psicológico y lo religioso.

Nuestra intención es preguntarnos por lo que ocurre con algunos participantes de un movimiento de espiritualidad de origen indio, con fuerte diálogo con las sensibilidades estilo Nueva Era, denominado El Arte de Vivir (EADV). Nuestro objetivo es analizar su circulación entre el movimiento y otras prácticas consideradas "terapéuticas" para mostrar la existencia de un circuito común amparado en principios ordenadores como el bienestar, el equilibrio y un particular vínculo con la energía.

Creemos que indagar en esa dimensión puede ayudar a acercar más lo vivido y las mediaciones que tejen las personas en su día a día y como allí se construyen modos específicos de pensar y vivir la propia acción espiritual, modos que no pueden ser reducidos a la perspectiva universalista de la acción estratégica y la lucha por un posicionamiento. Ello nos lleva a privilegiar los contextos prácticos de producción de la espiritualidad como un proceso que no puede ser asociado exclusivamente a una cosmología sistemática específica, una tecnología, un saber o un grupo, tampoco a una experiencia individual, sino a una multiplicidad de espacios y recursos que se complementan, se articulan y se negocian.

Luego de reconstruir algunos puntos centrales del debate contemporáneo sobre la espiritualidad, nos centramos en el discurso institucional del movimiento y su localización en un régimen de disputas públicas en tensión entre lo terapéutico y lo religioso. Finalmente, analizamos un régimen de relaciones cotidiano que produce agenciamientos múltiples y una gramática alternativa en una red más amplia a su lógica institucional y su intervención pública sobre el bienestar. Entendemos que tal articulación puede pensarse en un proceso más amplio de cotidianización de los saberes, las técnicas y las prácticas que conlleva una hibridización de las fronteras, tal vez nunca demasiado estables, entre los órdenes corporales y anímicos. Un proceso que, contra la idea de una sociedad del riesgo permanente, algunos analistas han denominado una sociedad de soluciones múltiples o "democracia técnica"(1).

\section{El arte de vivir ¡terapia o religión?}

A principios de la década de 1980 el gurú indio Ravi Shankar organiza en California la Fundación El Arte de Vivir. Esta se basa en una copiosa oferta de seminarios, talleres de respiración, meditación y comida natural. Heredero de los movimientos de transnacionalización espiritual que emergieron luego de la descolonización de India, el movimiento liderado por Ravi Shankar reformula una serie de nociones y prácticas hindúes tradicionales centradas en el bienestar personal y la transformación social adaptadas a un público occidental $^{(2,3)}$. La ideología de la Fundación El Arte de Vivir promueve un cambio de vida, una experiencia del confort personal y una práctica que apunta a alcanzar "la felicidad aquí y ahora". En ese horizonte, el movimiento guiado por Ravi Shankar difunde un 
mensaje de paz y transformación social a nivel global apoyado en un marcado énfasis en el equilibrio personal entre lo espiritual y lo mundano que, si bien puede ser identificado con la tradición hindú en una versión occidental y globalizada, también puede ser identificado en el horizonte de la espiritualidad contemporánea, según sea el caso, a partir de su presencia en la amplia sensibilidad Nueva Era o en los recursos terapéuticos de lo "alternativo".

La presencia pública en Argentina ha llamado la atención de algunos investigadores. Algunos trabajos se han centrado específicamente en sus aspectos terapéuticos. Por ejemplo, Funes ${ }^{(4)}$ analiza la categoría y la gestión del estrés como una categoría central de sus concepciones del bienestar y el malestar y D'Angelo ha descripto el lugar de sus técnicas corporales ${ }^{(5)}$. Otros trabajos se han preocupado por las articulaciones con otros ámbitos que exceden al propio movimiento, sobre todo los que tienen que ver con la vida empresarial y política. Vargas y Viotti ${ }^{(6)}$ analizan algunas de sus prácticas en eventos masivos y su sincronicidad con discursos empresariales del emprendedorismo; Viotti ${ }^{(7)}$ analizó su impacto mediático y su inserción en las narrativas católico-seculares dominantes del espacio público en Argentina en función de su presencia en una zona culturalmente legítima y socialmente dominante; y Viotti y Funes ${ }^{(8)}$ se centraron en algunas relaciones con la vida política institucional y sus propios modos de entender la transformación personal, social y cósmica. Sin embargo, salvo el análisis de DÁngelo que analiza la relación con el campo psicológico ${ }^{(9)}$, poco se ha dicho sobre lo que el movimiento comparte con otras prácticas terapéuticas, sobre todo, con el horizonte de las Ilamadas "terapias alternativas".

Partimos de la hipótesis de que experiencias como la de la Fundación El Arte de Vivir han alterado los modos recientes de entender las fronteras entre lo religioso y lo terapéutico en zonas "centrales" de la sociedad argentina. Ese proceso se da en un marco mucho más amplio de difusión de la espiritualidad a nivel masivo, con presencia en lo laboral, económico, pedagógico y estético, que excede el estatuto "alternativo" con que fue caracterizado durante la década de 1980 en Latinoamérica ${ }^{(10)}$. Este proceso, lejos de suponer un proceso homogéneo, adquiere matices y singularidades regionales, nacionales y en función de grupos sociales, étnicos y de género. En Argentina, este movimiento viene adquiriendo una fuerte visibilidad pública y un despliegue capilar en diversos espacios sociales. Sobre todo, pero no exclusivamente, en el mundo urbano y en la sensibilidad cultural de sus sectores medios, clásicamente identificados como medicalizados, psicoanalizados o cercanos a una religiosidad secularizada.

Si hasta hace poco era posible ignorar la implicancia de la religiosidad en el bienestar, sobre todo con base en una idea restrictiva de lo religioso como sinónimo de una institución eclesial más o menos alejada de la religiosidad vivida, nos vemos obligados a repensar esas fronteras constantemente. Resta indagar si hasta hace poco esas fronteras eran realmente más estables o eran el resultado de nuestras herramientas de análisis, impregnadas de una agenda y de categorías poco sensibles al lenguaje de lo cotidiano y a los ensamblajes de la vida social. Sea como fuere, desde la presencia pública cada vez más central de la espiritualidad, sobre todo en su estilo Nueva Era pero también en sus versiones cristianas más convencionales como el carismatismo católico o el pentecostalismo protestante, vemos proliferar argumentos que promueven la cercanía y equilibrio entre lo íntimo y lo público, lo discursivo y lo material, lo anímico y lo corporal, entre la vida humana y el entorno ambiental, incluso muchas veces recurriendo a fuerzas no humanas. Esa presencia pública supone los ámbitos más diversos: el espacio urbano, los medios de comunicación, las escuelas, las empresas, las instituciones de encierro o los hospitales. Pareciera que el lenguaje del "holismo" amenaza con convertirse en un código de época.

¿Cómo dar cuanta de ese proceso sin reafirmar una frontera que nuestros análisis llevan implícitos? Aquí resulta relevante reflexionar hasta qué punto nuestras categorías son herederas de un proceso de "gran 
división" entre lo corporal y lo anímico que se enmarcan en un ordenamiento más amplio entre lo universal y dado, por oposición a lo singular y construido que reverbera en las tensiones modernas entre naturaleza/cultura o social/individual ${ }^{(11,12,13)}$.

Más allá de la eficacia social de esa separación en la vida social, el problema persiste en tanto existe una "desproporción en las escalas comparativas" que utilizamos analíticamente como antropólogos, sociólogos o historiadores. Como señalan Stolze Lima y Goldman $^{(11)}$ esa es una de las dificultades de comparar, en la medida que en ese ejercicio suelen hacerse recortes descontextualizados y suelen tomarse como unidades de contraste que corresponden a planos diferentes.

Por ejemplo, cuando se contrastan datos que emergieron de una descripción bien localizada en el trato cotidiano con opiniones o posiciones que emergen en el discurso de los medios masivos o intervenciones públicas normativas dichas o escritas, se corre el riesgo de suponer fenómenos diferentes y no dos caras del mismo. Ello puede llevar a suponer, por ejemplo, que la frontera entre lo secular y lo sagrado es estricta en las religiosidades "institucionales" y más lábil en las "no institucionales" o, aun, que existe una diferencia entre los "grupos religiosos o espirituales" y las "redes terapéuticas alternativas", cuando en realidad la diferencia es de énfasis y no del proceso en sí. Lo que nos llevaría a pensar que la flexibilidad de las fronteras entre lo sagrado y lo secular y la red de las relaciones entre personas, cosas y fuerzas no naturales es un problema de perspectiva y no de un proceso en sí. Creemos que tal vez un ajuste en ese tipo de enfoque pueda, incluso, ayudarnos a hacer mejores comparaciones.

\section{La espiritualidad: una mirada desde lo cotidiano}

Los estudios sobre la espiritualidad contemporánea muestran una gran heterogeneidad en años recientes. Si tuviésemos que agruparlos en diferentes abordajes, podríamos señalar al menos tres corrientes.
Un primer grupo plantea una descripción sociológica e histórica más o menos general, subsumida a la narrativa moderna de la individualización, que supone una pérdida de un horizonte heteronormativo de la religión y la emergencia -por vía de los movimientos espirituales- de un modelo autónomo o individualista $^{(14,15,16)}$. Si bien estos trabajos tienen el mérito de una lectura de largo plazo, se han mantenido en un nivel muy ideacional $y$, como consecuencia, muchas veces se han centrado excesivamente en la tensión entre un orden cohesivo, propio de una religiosidad eclesial, y una concepción del individualismo, propio de la espiritualidad, que supondría una subjetividad infrasocial. Este tipo de análisis posee algunas dificultades para detectar la capilaridad de las experiencias autopercibidas como espirituales en los más diversos ámbitos y particularmente para entender su presencia y difusión pública contemporánea.

Un segundo grupo ha desarrollado una crítica a esos trabajos pioneros, mostrando cómo el foco en la vida cotidiana permite reconstruir relaciones sociales y cuestionar el excesivo énfasis que esos trabajos le dieron a la autonomía individual. En ese sentido, Wood $^{(17)}$ y Wood y Bunn ${ }^{(18)}$ se preocupan por el problema de la autoridad, a partir de una relectura de la teoría de la práctica social de autores como Pierre Bourdieu y Michel Foucault. Estos autores señalan que es necesario repensar la dicotomía entre autonomía individual y autoridad externa y subrayan que existen autoridades múltiples en las prácticas espirituales estilo Nueva Era, como líderes, especialistas y fuerzas no naturales, que influyen sobre los grupos e individuos de manera profunda y con un fuerte nivel de estabilidad. Además, muestran cómo esas prácticas y esas autoridades múltiples se vinculan con procesos de producción y reproducción de las clases sociales, particularmente, los grupos identificados como sectores medios. Esos autores priorizan las redes y las prácticas cotidianas, describiendo las tramas relacionales, con su productividad social y cosmológica, en un movimiento que matiza algunos dualismos implícitos en la sociología de la 
modernización religiosa: social/individual, sagrado/profano y alma/cuerpo.

En último lugar, desde una perspectiva más radicalmente pragmática, Bender y McRoberts ${ }^{(19)}$ han insistido en una crítica semejante a los estudios más clásicos que entienden la espiritualidad como un fenómeno independiente de lo religioso y caracterizado exclusivamente por la individualización y la privatización. Por el contrario, estos autores muestran hasta qué punto la espiritualidad posee una génesis histórica y una fuerte dimensión relacional -no solo un énfasis en la "interioridad" - y de qué manera es un recurso performativo con consecuencias públicas. Este último aspecto resulta crucial porque, contra la idea de la privatización de la religión, muestra cómo la espiritualidad moviliza formas diversas de acción pública y de adhesiones políticas en las sociedades contemporáneas de la mano de los modos de mediación novedosos que implican los recursos de la comunicación de masas y la industria cultural.

Estos trabajos sobre la espiritualidad contemporánea son un recurso útil para repensar las distinciones entre lo estrictamente religioso y lo terapéutico, en la medida en que dejan parcialmente de lado las distinciones más estrictas entre esferas o campos independientes, o incluso su redefinición contemporánea, para centrarse en la vida cotidiana. Asimismo, descentran el foco de los especialistas y las intervenciones públicas y dan un nuevo estatuto epistemológico a los espacios más "banales" e "impuros". Por último, permiten también suspender los recortes más convencionales entre grupos religiosos o terapias, como si fueran una realidad en sí misma.

Ese movimiento reciente posee en América Latina desarrollos propios que sería muy extenso reconstruir aquí. Al menos en Argentina, el análisis de lo que aquí estamos denominando y recortando analíticamente como bienestar espiritual encuentra una serie de trabajos y reflexiones que han crecido en las últimas décadas, de la mano de una problemática que ha adquirido cada vez más visibilidad pública. Aunque priorizaron la lógica institucional, tanto los trabajos centrados en movimientos o grupos religiosos y/o espirituales mostraron una tendencia a la articulación ${ }^{(4,5,9,20)}$. Estos trabajos han abordado el problema de la articulación entre lo médico-psicológico y lo espiritual, y han tenido un particular desarrollo, sobre todo, bajo el concepto de "terapias alternativas". Tanto desde el punto de vista de la construcción y negociación de las identidades profesionales de la medicina y la psicología ${ }^{(21)}$ como desde el punto de vista más englobante de especialistas y frecuentadores de prácticas "alternativas" como la reflexología, el yoga y la medicina ayurveda, que suponen diferentes grados de articulación con algunos principios no naturalistas en el horizonte de un "pluralismo terapéutico" que se caracteriza por la “elección" entre opciones diversas ${ }^{(9,22,23,24,25)}$.

Si los primeros se centran en un análisis de la incorporación de lo espiritual en lo médico-psicológico, con foco en la oferta, los segundos se concentran en la adaptación de prácticas-que, en principio, pertenecían a un horizonte cosmológico- a los regímenes seculares de gestión de la salud, no sin tensiones, resignificaciones y persistencias. Aun manteniéndose en la "esfera" de las denominadas terapias alternativas, estos trabajos poseen un enorme valor y han ampliado el conocimiento de una serie de prácticas que manifiestan regímenes de causalidad y de eficacia no naturalista, llegando incluso a cuestionarse los límites o las fronteras de lo estrictamente "terapéutico" y mostrando diferentes grados de vínculos con esas tecnologías y saberes que van desde el uso estratégico hasta la adhesión a sus principios básicos.

Si desde un punto de vista esa separación puede ser socialmente eficaz, desde otro no posee una relevancia tan sustantiva. Es, en parte, el lenguaje del que disponemos el que queda atrapado en la tensión entre lo religioso-espiritual y lo médico-terapéutico. $\mathrm{Y}$, como es bien sabido su capacidad performativa, recorta nuestros objetos de estudio, nuestros temas y nuestros problemas de un modo singular. Desde esa premisa, señalo tres focos de separación que pueden ser repensados desde un análisis sobre la vida 
cotidiana y los diferentes agenciamientos plurales del bienestar espiritual.

En primer lugar, el recorte que realizamos entre "terapias alternativas" y "grupos religiosos o espirituales". Mientras el primero tiende a un diálogo en el horizonte de los estudios sobre salud, el segundo es incorporado a las ciencias sociales de la religión. El trabajo de María Julia Carozzi ${ }^{(26)}$, desde el título que alerta sobre la posibilidad de leer esos procesos en su simultaneidad y la descripción de la red como lógica morfológica y señalamiento de algunos principios básicos como la cosmización, la autonomía y la desjerarquización, se preocupó por no separar esos ámbitos en esferas independientes; $y$, aunque algunos de sus principios o su morfología no hayan servido para todos los casos particulares -sobre todo cuando se puso el énfasis en los discursos públicos y los principios totalizantes de grupos espirituales o terapéuticos- creemos que su descripción todavía es útil al priorizar el orden cotidiano y la circularidad de los frecuentadores.

Particularmente, cuando analizamos los discursos y las prácticas públicas surge un segundo punto de distanciamiento. Sobre todo, cuando el foco está puesto en los especialistas y las intervenciones de colectivos específicos, los analistas tendemos a concentrarnos en los modos de legitimación, de diferenciación, de heterodoxia y/o de resistencia, porque muchas veces esto es lo que aparece en la interacción con sus líderes, referentes o expertos. De ese modo, la articulación entre esos términos aparece como un "híbrido", en el sentido que Bruno Latour ${ }^{(12)}$ entiende el término, como una combinación de dos elementos que se enuncian separados pero se viven como unidos. Asimismo, a partir de ellos, accedemos habitualmente a perspectivas sistematizadas y relativamente coherentes y creemos constatar algún tipo de sistema con principios, valores y prácticas organizados como si fueran exclusivas de los grupos que analizamos y no parte de una gramática que se construye en un proceso más amplio.

En tercero y último lugar, incluso cuando nos centramos en los discursos menos públicos, sobre todo cuando damos por sentado un sujeto individual como interlocutor válido y locus único de la interacción, tendemos a reconstruir sus "representaciones" y sus trayectorias (terapéuticas y/o religioso-espirituales) de modo aislado, dando por sentada una concepción de la persona que aparece como universal pero que se asemeja bastante al modelo del individualismo moderno, que supone un agente que "selecciona" y "elige" en un mercado o un escenario "plural" de bienes de salvación o bienestar. El foco puesto en la elección individual corre el riesgo de dar por sentada una concepción de la persona estrictamente individualista, sin indagar en el modo de subjetividad que allí se produce y que creemos supone elementos diferenciados con el modelo clásico del individuo ${ }^{(27)}$. Buena parte de la bibliografía de las ciencias sociales se ha centrado en la imagen del "buscador", dando por sentado un tipo de individuo demasiado asociado con individualismo moderno, borrando en muchos casos las tramas de relaciones sociales que producen esas "elecciones" o las propias teorías que esos agentes tienen sobre su propia persona, las que muchas veces no se corresponden con un agente que actúa en el mundo de modo tan libre. Un enfoque muchas veces sobredimensionado por el propio carácter de nuestros abordajes teórico-metodológicos como, por ejemplo, el uso exclusivo de la entrevista en profundidad que puede centrarse unilateralmente en la "experiencia individual"(8).

Retomando algunos de los planteos de un enfoque relacional de lo que hace a la subjetividad en el horizonte de la espiritualidad contemporánea, queremos argumentar que una mirada sobre lo cotidiano que asuma seriamente algunos aspectos vividos como reales por sus frecuentadores puede ayudarnos a recomponer un cuadro algo más complejo de este proceso. En cierto sentido, nos interesa restituir el análisis relacional descripto por los primeros trabajos que, en la región, describieron la Nueva Era y las terapias alternativas como parte de una misma red, y extenderlo más allá hacia una trama más amplia que incorpore lo cotidiano y los regímenes de subjetivación. 
Entendemos la espiritualidad como una categoría que evidentemente no es novedosa, pero que ha adquirido una renovada presencia contemporánea, que existe como puesta en acto, es decir que existe en la medida en que es actuada, con gran capacidad de circulación y performatividad en los más diversos ámbitos ${ }^{(19,28,29)}$. Al mismo tiempo, entendemos que esa pragmática de la espiritualidad supone una gramática heterogénea, pero no por ello infinita y sin especificidad, que incorpora una concepción no naturalista de la causa y la eficacia, un orden cosmológico relacional y un trabajo sobre uno mismo y que se manifiesta en intensidades diversas que se basan en mediadores materiales y discursivos de diferentes linajes: esoterismos varios con una presencia consolidada durante el siglo $\mathrm{XX}$, la cultura self-help y tecnologías de la subjetividad de origen oriental, incluso algunas prácticas amerindias, adaptadas a la vida cotidiana de colectivos urbanos de las sociedades euroamericanas contemporáneas ${ }^{(30,31)}$.

Desde el punto de vista de la circulación cotidiana de esos saberes y prácticas, es posible que la tensión entre lo terapéutico y lo religioso/espiritual, e incluso sus disputas por la legitimidad en un campo o las perspectivas sistémicas que emergen cuando el foco está puesto en los discursos públicos, adquiera otro matiz. No solo es mucho más difícil recortar las esferas autónomas de lo terapéutico y lo espiritual, sino que incluso la propia espiritualidad supone una trama de circuitos, de sentidos y de prácticas diferenciadas. Como veremos más adelante, si bien desde el discurso público se insiste en las ventajas físicas, médicas e incluso psicológicas de la meditación, sobrevalorando su función en el bienestar entendido como un proceso biológico y psicoemocional, desde la vida cotidiana, las causas de ese bienestar exceden por mucho una definición biológica y sociopsicológica de la subjetividad, incorporando una dimensión no humana como la energía que supone una fuerza vital transformadora que puede producir malestar cuando no circula correctamente y bienestar cuando se logra canalizar y equilibrar.

La vida cotidiana de los actores en acción y la complejidad de lo que $\operatorname{Law}^{(13)}$ ha denominado "formas no convencionales", supone procesos de circulación y ensamblajes que pueden ayudarnos a repensar los puentes y articulaciones diversas entre el mundo médico-psicológico, las terapias alternativas y la espiritualidad Nueva Era. En ese sentido, nos parece importante el análisis de Law ${ }^{(13)}$ sobre los "ensamblajes" en contextos religiosos donde resulta significativo dar una perspectiva realista que permita ampliar la realidad de las personas que la viven y, en ese proceso, indagar incluso la noción misma de la persona que está en juego. Tal abordaje podría leer también críticamente la perspectiva del individualismo metodológico que se concentra en los usuarios o frecuentadores de prácticas de ese tipo y sus "elecciones terapéuticas" o sus "elecciones espirituales" como "campos" o "esferas" de acción diferenciadas.

\section{ASPECTOS METODOLÓGICOS}

El material proviene de observación participante, entrevistas abiertas y convivencia con participantes del movimiento El Arte de Vivir y, sobre todo, del acompañamiento de sus seguidores más allá de las actividades en el propio movimiento, sus relaciones con su entorno personal, sus lecturas y la trama de mediaciones que configuran su vida cotidiana. Además, se conformó una base de datos secundarios con material periodístico de diversos medios de comunicación, con la intención de analizar las intervenciones públicas de la Fundación y también las reacciones en ese ámbito de otros actores sociales.

Las entrevistas y la observación se realizaron desde 2012 hasta 2017, de modo continuo, en diferentes ámbitos y contextos. En todos los casos, se explicitó el propósito y el alcance de la investigación y se garantizó, salvo voluntad explícita, el anonimato de los interlocutores. El trabajo total cuenta con treinta entrevistas registradas digitalmente realizadas a referentes, cuadros intermedios y, sobre todo, a participantes ocasionales de las actividades de la Fundación. Este trabajo se complementa con registros de campo de 
conversaciones e intercambios verbales informales, recopilados en el diario de campo. La observación participante se realizó en talleres y cursos de diferente índole llevados a cabo en días específicos de la semana en dos sedes de la Fundación situadas en los barrios de Colegiales y Villa Crespo de la Ciudad Autónoma de Buenos Aires. También se ha realizado observación en diferentes retiros en la localidad de Tigre, provincia de Buenos Aires; en conferencias sobre temas específicos o con invitados especiales, referentes internacionales o el propio Ravi Shankar; y en intervenciones o debates en espacios públicos que la Fundación desarrolla en ámbitos específicos donde tiene injerencia (hospitales, cárceles, vía pública). Finalmente, he participado de casi todos los eventos de meditación colectiva realizados cada año en plazas públicas de la Ciudad Autónoma de Buenos Aires denominados "Argentina Medita", en los que he realizado además una encuesta con los participantes sobre su perfil social, de estilo de vida y sus recurrencias espirituales, religiosas y terapéuticas. Tanto las entrevistas como los registros de campo de conversaciones $u$ observaciones han sido codificados en función de temáticas recurrentes que emergieron en la investigación.

\section{LA DISPUTA PÚBLICA POR LA SALUD}

Algunas de las intervenciones públicas de referentes de El Arte de Vivir pueden darnos una idea de cómo la espiritualidad se monta sobre argumentos científicos que suponen que la cura puede ser medida en términos de eficacia "corporal". Eugenio, uno de los coordinadores de los cursos y talleres ofrecidos semanalmente por la Fundación señalaba, en una de las charlas introductorias, lo siguiente:

La meditación mejora la organización celular, está comprobado por un montón de estudios médicos. Retrasa el envejecimiento y mejora la calidad de vida porque rejuvenece las células. Más allá de eso está muy comprobado que quien medita y se alimenta saludablemente tiene menos posibilidades de enfermarse de cáncer y tener un ataque cardíaco, menos chances de deprimirse o tener estrés. La respiración aumenta la oxigenación y eso transforma todo el metabolismo del cuerpo y eso tiene consecuencias en la vida de las personas, en el carácter, en las relaciones. Hay una base biológica en todo esto. (Eugenio)

El propio Ravi Shankar, en su visita en 2012 a Argentina, en incontables espacios manifestó que la respiración es una técnica que ayuda a liberarse del estrés y mejora la calidad de vida y que está "estudiada científicamente" su eficacia como complemento de otras técnicas médicas y psicológicas. En una conferencia pública en el hotel Sheraton de la Ciudad de Buenos Aires, por ejemplo, rodeado de periodistas y seguidores, señaló que las técnicas de respiración promovidas por El Arte de Vivir ayudan también a prevenir las "enfermedades mentales de estos tiempos como la depresión y la ansiedad".

En otra de sus apariciones púbicas señaló que el cáncer puede tener causas biológicas, pero también asociadas con la alimentación y el estilo de vida y que las técnicas de meditación y una vida saludable pueden ayudar a revertir esos procesos. En una entrevista realizada en el diario Clarín titulada "La mente y la respiración tienen un rol muy importante para curar el cuerpo"(32), Shankar afirmaba que:

Sí, lo hemos experimentado en las últimas décadas con miles y miles de personas que han encontrado la cura a través de su propia respiración. La mente y la respiración tienen un rol muy importante para curar el cuerpo. Si las personas enfermas no descansan, por más que reciban medicación no se van a curar. Nuestro cuerpo tiene una habilidad natural de curarse cuando tiene descanso profundo, y la mente puede otorgar ese descanso al cuerpo. ${ }^{(32)}$

Por supuesto que existieron reacciones abundantes en los medios de comunicación, 
en una nota publicada en el diario Clarín "Todo tratamiento contra el cáncer debe tener un soporte científico"(33), un médico oncólogo, vicepresidente de la Asociación Argentina de Cancerología, subrayó la falta de evidencia empírica de que las "terapias alternativas" curasen el cáncer y que este debía ser tratado biológicamente. Haciéndose eco de que una reacción mucho más amplia no dejaba de mencionar la complementariedad de esas técnicas y el beneficio de una dieta y el trabajo de meditación como un factor que mejoraba la calidad de vida:

Existe la certeza de que el tratamiento multidisciplinario es la mejor forma de abordar la enfermedad. También debemos sostener que todo lo que permita mejorar la calidad de vida -como una dieta saludable o las técnicas que optimizan el estado de ánimo- ayudan en el proceso hacia la retracción de la enfermedad. Pero todo esto se debe concretar dentro de un contexto de asistencia con soporte científico. ${ }^{(33)}$

Lo que resulta interesante es que los referentes del movimiento e incluso algunos de sus impugnadores médicos sostenían que existe una complementariedad entre ambas técnicas corporales y espirituales. ¿Pero qué idea de lo espiritual movilizaban? En esas intervenciones, la dimensión terapéutica de la espiritualidad se mantenía dentro de criterios complementarios y dentro de un horizonte en el que las causas del bienestar y el malestar son en primer lugar naturales, o sea, vinculadas a fenómenos corporales, físicos y biológicos $y$, en segundo lugar, psicosociales, es decir, asociados con estilos de vida y conductas que tienen que ver con el cuidado de uno mismo y su entorno.

¿Qué quería decir el instructor y el propio Ravi Shankar con sus afirmaciones? ¿Podemos afirmar que esas concepciones son las únicas que circulan entre los referentes y los seguidores de El Arte de Vivir? La concepción de que lo corporal es medible y cuantificable en términos de eficacia aparece como un discurso público de legitimación, en el que lo espiritual es totalmente compatible con lo médico-psicológico. Esa concepción circula ampliamente en discursos públicos y también cotidianos. Como decía un seguidor de Shankar en una conversación informal que registré en mi diario de campo:

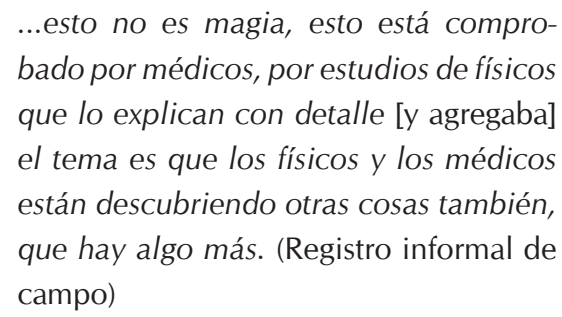

Nos interesa ahora indagar un poco en ese "algo más" en la medida en que, desde otro punto de vista menos evidente en las manifestaciones públicas, el bienestar supone una trama más extensa que la del propio cuerpo.

\section{La trama espiritual y la ordinarización del bienestar}

Algunos momentos compartidos con Joaquín y Julia, dos seguidores de El Arte de Vivir en Buenos Aires que se definen como personas "espirituales" o con una "búsqueda espiritual", puede ayudarnos a poner en foco lo que se entiende aquí como una participación plural en la espiritualidad contemporánea y como parte del mismo régimen de bienestar que supone diferentes recursos y tecnologías que exceden a un "grupo" o a una "terapia" y que conforman un espacio de interlocución común no reducible a ninguno de esos términos.

Joaquín es economista, apenas pasó los cuarenta años y es egresado de la Universidad de Buenos Aires. Compartí con él actividades variadas dentro y fuera de la Fundación. Si su identificación personal con EI Arte de Vivir era intensa y comprometida, no solo había participado de varios cursos y empezado a formarse como instructor, sino que se comprometía intensamente en actividades en las cárceles y en los programas de asistencia a las personas que viven en la calle, vivía esa identificación como un camino entre otros. Un lugar donde él había encontrado 
algo que se manifestaba en muchos otros "caminos espirituales" y que aparecía en muchas otras personas que fue encontrando y quienes tenían su propio recorrido hacia lo que él consideraba "el mismo fin", es decir, el "crecimiento personal", el "descubrirse a uno mismo" y el "estar en equilibrio".

Julia apenas tiene algo más de treinta años, estudió psicología y me contó infinidad de veces detalles de su familia judía no practicante, de quienes siempre se sintió muy cerca y a quienes los quiere convencer de participar en El Arte de Vivir. Si bien siempre se sintió "lejos de la religión" insiste en que a partir de su participación en la fundación está aprendiendo a revalorizar muchas cosas de la "espiritualidad judía" que había dejado de lado, detallando, al igual que Joaquín, que la espiritualidad es una y que cada uno la recorre por caminos inesperados.

Conocí a Joaquín y a Julia en un curso de la fundación hace más de cinco años. En ese curso inicial fue donde aprendimos una de las técnicas más importantes de El Arte de Vivir. Era uno de los primeros cursos que tomaba y ambos estaban ya insertados en actividades diversas de la fundación. Luego de algunos días de prácticas conjuntas de yoga, meditación y de oír charlas sobre alimentación y vida sana, llegó el momento final y más esperado, la práctica de lo que se considera el momento más especial de los cursos: el Sudarshan Kriya.

El término puede traducirse como "visión apropiada de purificación", técnica revelada al propio Ravi Shankar durante la década de 1980 en California y que consiste en ejercicios de hiperventilación intensa, acompañados de movimientos de brazos de modo rítmico y que finaliza con una quietud corporal que habitualmente produce estados de fuerte conmoción personal que llevan tanto al Ilanto como a la risa y a visualizaciones o sensaciones de éxtasis intenso. Versiones cortas de ese Kriya o "práctica de purificación" son aprendidos por los participantes para realizarlo en sus propias casas de modo habitual y constituyen uno de los ejes de la manutención de un estado de bienestar que se acompaña por el respeto de una dieta rigurosa y el consumo de agua en grandes cantidades.

Este es un proceso importante en la socialidad de la Fundación y un hito del efecto que muchos participantes ocasionales vivencian como "transformador". Esta técnica es un proceso que ha sido detallado por otros autores mostrando sus manifestaciones "somáticas" como por ejemplo por D'Angelo ${ }^{(5)}$.

Esta experiencia fue central para ambos. De allí en más, ambos participaron activamente en los grupos y en la fundación. Para Julia es un "trabajo con uno mismo permanente, un trabajo de purificación y de equilibrarse". Para Joaquín es:

\section{...lo más importante que aprender como técnica, es el punto de partida de todo lo que viene después porque te produce tal satisfacción que luego querés volver a eso todo el tiempo. Es una movilización energética profunda, como que conocés un poquito la divinidad. (Joaquín)}

Esta tecnología que combina respiración, movimientos corporales y meditación, pero a su vez la movilización de lo que en la puesta en acción supone una "movilización energética profunda". El tema de la movilización energética resulta sustancial en la medida que incorpora a la eficacia en el bienestar que esta técnica proporciona a los iniciados un elemento extrahumano que funciona como una fuerza que debe ser descubierta y gestionada por los recién Ilegados a los cursos y, más adelante, por los iniciados. Además, como señalaba Joaquín, esa energía puede ser entendida como divinidad, mostrando sin duda aspectos de los modos en que en El Arte de Vivir esa experiencia es enmarcada en una concepción más amplia de lo no-humano. Este recurso, además, proporciona un estado de bienestar personal o una "borrachera" que muchos de los seguidores identifican informalmente como estar empranado, o liberar mucho prana, que es uno de los modos utilizados para referirse a la energía en sentido amplio.

Sobre este aspecto de la práctica de los Kryas, asociado además a la alimentación y 
la meditación, como categoría más amplia y en su sentido moral de la vida servicial propuesta por El Arte de Vivir, cabe señalar dos cosas. En primer lugar, si bien en los discursos públicos de la fundación se insiste en la dimensión "empíricamente comprobada" y la "evidencia científica" de las bondades de estas técnicas para la salud, las personas que la utilizan van mucho más allá de esas definiciones naturalistas de la causa del bienestar. Se movilizan fuerzas que no podrían estar en un argumento cientificista que se base únicamente en el dato estadístico o en los estudios de eficacia referidos constantemente por la fundación en sus intervenciones públicas. En segundo lugar, si bien el término prana, utilizado por los seguidores más frecuentes de las actividades de la fundación como Julia y Joaquín, supone sofisticadas elaboraciones en la tradición hindú, las personas con las que compartí bastante tiempo solo excepcionalmente se referían a su definición intelectual, en general la usaban como sinónimo de "energía" y, como veremos a continuación, se entroncaba en un horizonte mucho más vasto que el de la propia fundación.

Dos años después de haber participado con Julia y Joaquín de ese curso, visité a Joaquín en su casa del barrio de Belgrano. En su casa vendía miel artesanal y tenía una pequeña biblioteca con títulos famosos de la Ilamada literatura de autoayuda: El alquimista de Paulo Coelho, La enfermedad como camino de Thorwald Dethlefsen y Rudiger Dahlke, el Libro tibetano de los muertos y manuales de comida vegetariana. Su casa tenía varios ramos de flores y un pequeño altar con la imagen de Ravi Shankar. Apenas lo reencontré, me contó que venía de una sesión de reiki y que estaba totalmente "conectado". Le pregunté cómo entendía la práctica del reiki como una "terapia alternativa" al mismo tiempo que participaba de las actividades de la fundación, sobre todo, teniendo que cuenta que el reiki es un recurso de "equilibrar la energía" que, aunque supone una amplia difusión en Buenos Aires, proviene de una tradición de origen japonesa iniciada por el monje del budismo zen Mikao Usui muy alejada del neo-hinduismo que propone Ravi
Shankar. Julián desestimó mi comentario y me explicó que, en el fondo, toda la energía es la misma y que hay que buscarla de diferentes maneras:

Yo voy a reiki de vez en cuando, me hace bien. ¿Vos me decís que es una terapia alternativa? Yo te digo que no, que es una práctica que te reconstruye energéticamente que es totalmente complementaria con lo de "El Arte". Uno va probando y se va quedando con las cosas que le hacen bien, para mí son dos modos de lo mismo, de ayudarme a estar mejor conmigo mismo y con el mundo a mi alrededor, son cosas que te hacen más consciente. No es algo terapéutico, hay gente que se acerca porque tiene algún problema de salud a estas prácticas, pero eso le hace cambiar de vida, después se dan cuenta que la salud es una consecuencia de otros procesos que tienen que ver con mover la energía, que siempre es la misma. Al final son prácticas, todas, que te hacer estar bien, yo a la larga no diría que es una terapia, lo de la terapia lo deja chico. (Julián)

Con este comentario, Julián desarmaba mi propia sugerencia de la diferencia entre El Arte de Vivir y el reiki, entre una práctica que me parecía más institucional y otra más "alternativa", mostrándome que para su propio régimen de construcción espiritual del bienestar ambas estaban en sincronía. Al mismo tiempo, deslegitimaba mi propia diferenciación entre lo espiritual y lo terapéutico, señalando que la concepción de alguna de esas prácticas como "terapia" eran poco menos que parcial y que la "energía" que se ponía en movimiento era la misma.

Poco tiempo después reencontré a Julia en una charla de la fundación, como era muy cercana a otras personas en común que conocía desde hacía tiempo, me invitó a su cumpleaños que era esa misma tarde. No había alcohol y toda la comida era vegetariana. En un momento de la tarde Julia se puso a charlar con unos amigos sobre una psicóloga que estaba consultando que trabajaba con 
los registros akáshicos. La "apertura de los registros akáshicos" es una práctica bastante difundida entre los frecuentadores de prácticas oraculares y que tienen un antecedente en el esoterismo europeo del siglo XX de la mano de la teósofa británica Annie Besant. De todos modos, la presencia actual poco tiene que ver con esa matriz esotérica intelectual y supone una amplia difusión en versiones mucho más articuladas con la psicología positiva y la autoayuda. Las consultas presuponen que el/la especialista funciona como médium de entidades que poseen información personal del consultante y que, una vez abiertos esos registros, ese canal de comunicación funciona como un recurso de autoconocimiento y de reconexión con el "éter" o energía vital donde existe un registro personal.

La consulta frecuente de Julia a esta especialista en registros akáshicos me llamó la atención de un modo parecido al que me produjo la visita de Julián al reikista. Lo interesante es que muchos de los participantes de El Arte de Vivir presentes se mostraron muy interesados en la práctica y hasta pidieron que Julia les pasara el contacto para poder hacer sus propias consultas. En un momento, el episodio produjo una breve charla sobre la existencia de diversos caminos para acceder a la "energía del Universo" o "lo que hay escondido adentro de nosotros mismos". Para todos ellos, la existencia de una fuerza vital era lo más importante. Más allá de que consideraran la importancia de las enseñanzas del gurú Ravi Shankar como un "camino que aceleraba el proceso" de acercamiento a esa fuerza, su foco estaba puesto en conectarse de la forma que fuere. Incluso Ravi Shankar, me decían, sostenía que los caminos para llegar a esa conexión son diversos, aunque aclaraba que tener un gurú ayuda a no perderse en un sinnúmero de alternativas. ¿Cuán extraño era que frecuentadores asiduos de un movimiento como El Arte de Vivir circularan por otros recursos espirituales de bienestar? ¿Qué diferencias había en sus propias definiciones de un movimiento espiritual y prácticas específicas? ¿Qué elementos en común permitían esa circulación y la asociación con una misma fuente vital?
Es claro que no todos consideran complementarias la participación en El Arte de Vivir, sobre todo cuando hay un grado de compromiso intenso, con prácticas específicas como el reiki o la consulta de los registros akáshicos. Como señalaba Joaquín: "El Arte te da un camino, enseñanzas, un espacio que te permite crecer", es decir, existe plena conciencia de que la dimensión del movimiento produce un espacio de pertenencia mucho mayor a una práctica de crecimiento personal. Sin embargo, la posibilidad de la circulación y la práctica específica de otras técnicas que desde una perspectiva podría incluirse dentro de las terapias alternativas, como el reiki, o prácticas oraculares muy específicas como los registros akáshicos, estaban a la orden del día.

Creemos que esa recurrencia no se explica solamente por la "elección individual" de Joaquín o de Julia, ni tampoco por la disposición de una "cultura espiritual" específica, aunque no podemos descartar una dimensión de la propia elección ni tampoco una regularidad cultural mayor que es el resultado de una serie de prácticas específicas. En cierto sentido, resulta importante subrayar una dimensión que puede matizar la polaridad de esos análisis, sin descontarlos del todo. Pero nos parece importante incluir una dimensión que radicalice el lugar de la energía como una fuerza ordenadora de esa trama de relaciones y de prácticas entre lo anímico, lo corporal y lo energético que nos ayude a entender qué forma adquiere la subjetividad allí cuando tomamos muy en serio los modos en que las personas involucradas se entienden a sí mismas. Una subjetividad que, tal vez, no pueda considerarse únicamente como la continuidad de un individuo que elige libremente ni tampoco como el producto de un proceso de socialización meramente humana ${ }^{(34)}$.

Según $\mathrm{Mol}^{(35)}$, una categoría médica ordinaria no supone un sentido específico o una pluralidad de sentidos sobre la base de una experiencia física y naturalmente universal sino una pluralidad de modos de hacerse en acto y, por lo tanto, una pluralidad de regímenes prácticos que la hacen real en sus consecuencias. Desde esta perspectiva, entendemos que 
todas esas prácticas en torno a la construcción del bienestar espiritual componen un circuito ordinario que conforma una multiplicidad de ensamblajes entre lo anímico, lo corporal y la energía. Ensamblajes diferentes, que van de prácticas como el Sudarshan Krya hasta el reiki y los registros akáshicos, cada uno de los cuales supone regímenes que sería necesario ampliar con más detalle para mostrar sus especificidades.

Ese circuito no es solamente un tránsito de individuos alrededor de "ofertas" de bienestar espiritual diferentes, identificadas como "religiosas" o "terapéuticas", sino un compuesto más amplio en donde si tomamos en serio las categorías de cuerpo, alma y energía que allí se movilizan, el propio concepto de individuo puede desmembrarse en regímenes de subjetivación que suponen una trama relacional más extensa. Una trama en donde la separación entre un fenómeno físico y universal no es el sustrato de "sentidos" diferentes socialmente construidos, sino un ensamblaje en el que tanto lo corporal como lo anímico y lo energético se enlazan en un régimen espiritual de bienestar.

Esos ensamblajes, sin embargo, también suponen una regularidad, movilizada por las mismas personas que ven allí un espacio de circulación y de prácticas comunes que apuntan a un fin semejante: buscar la conexión con uno mismo y con el cosmos. Pero es una regularidad resultante de un mapeo de esos circuitos y no impuesta por la lógica de un grupo o una terapia.

\section{CONCLUSIÓN}

No quedan dudas de que el mundo de la espiritualidad contemporánea es la punta de un iceberg en donde la cuestión del bienestar resulta central. En este trabajo hemos recorrido algunos aspectos característicos de los abordajes sobre ese proceso. El más importante nos parece la separación con que muchas veces se lo identifica, reconociendo el orden de lo terapéutico como relativamente independiente del orden de lo religioso o, en todo caso, viendo en estas prácticas un acercamiento entre dos esferas.

Como hemos intentado mostrar, este aspecto posee una importancia relativa en los discursos y las prácticas públicas, se perciben allí articulaciones y formas de legitimación que mantienen la autonomía relativa entre lo corporal y lo espiritual.

A partir de algunos frecuentadores de El Arte de Vivir pretendimos poner de manifiesto que indagar en la lógica cotidiana puede descentrar esa afirmación o, al menos, ordenar los términos de la comparación, diferenciando entre los discursos públicos y concepciones entramadas entre lo corporal y lo espiritual que presentan algunos desafíos.

$\mathrm{Si}$ en los discursos públicos la cientificidad o la evidencia son argumentos a favor de las prácticas que aparecen como corporales, un análisis de la circulación de las redes cotidianas muestra que la categoría de energía es un recurso que muestra una trama mucho más articulada entre lo corporal y lo espiritual que se aleja de las explicaciones naturalistas. La propia circulación de la energía da cuenta de un ensamblaje entre lo espiritual, lo anímico y lo corporal que solo puede recortarse en cada uno de esos términos a costa de dar una explicación parcial de las causas del bienestar.

La pista de la energía como elemento articulador de la dimensión cotidiana muestra una trama más compleja que hace a la sociomorfología y a los principios organizadores de los grupos y las prácticas terapéuticas como objeto de análisis en sí mismos. Desde este punto de vista existe un continuum que constituye un espacio de circulación que excede la lógica institucional de un grupo o una práctica y, al mismo tiempo, los principios que cada uno de ellos movilizan, sin desconocer las especificidades, pueden ser enlazados a partir de una gramática común que se extiende sobre los sistemas terminológicos y de pensamiento. Como vimos a partir de los seguidores de El Arte de Vivir y sus otros recursos para establecer una relación energética y espiritual equilibrada, estos se articulan en series múltiples a partir de una lingua franca centrada en la interioridad, la relacionabilidad y la cosmización del mundo. 
El caso analizado nos permitió sugerir una distancia entre los discursos y las prácticas públicas respecto de las cotidianas. A su vez, Ilamar la atención sobre la circulación entre espacios distintivos, pero en función de una gramática espiritual común. Ese énfasis, creemos, resulta una mirada necesaria en un contexto donde las prácticas definidas como espirituales se articulan con lo terapéutico. Frente a procesos que no siguen los modos tradicionales de definir lo religioso como una organización eclesial y un sistema de creencias organizado ni lo terapéutico como un espacio de saberes expertos médico-psicológicos resultan necesarios enfoques que se adapten al propio objeto en transformación.

Mostramos, entonces, la importancia de atender a una trama relacional y ensamblada que desafía el dualismo más convencional entre lo terapéutico/religioso. Por supuesto que, desde una perspectiva, esas fronteras existen y constituyen modos de delimitación institucional y espiritual, pero creemos que atender a la multiplicidad de los frecuentadores y aun a los modos en que su construcción del bienestar espiritual moviliza fuerzas, relaciones y artefactos más allá de esos "grupos" puede ser un recurso para pensar más allá de lo estrictamente religioso y más acá de lo estrictamente terapéutico.

Finalmente, creemos que ese rasgo de la espiritualidad contemporánea podría leerse en simultáneo con un proceso de ordinarización más general de los saberes, prácticas y técnicas utilizadas en la gestión contemporánea de la subjetividad. Si como mencionamos al principio de este trabajo algunos análisis contemporáneos se refieren a una "democracia técnica"(1), es decir, a una circulación ampliada de saberes expertos que al tiempo que producen hibridaciones permean espacios sociales no exclusivos, algo de ese proceso puede impactar también los saberes religiosos y terapéuticos. Por supuesto que este fenómeno no es del todo nuevo, pero su visibilidad contemporánea algo nos dice sobre su renovada presencia.

\section{REFERENCIAS BIBLIOGRÁFICAS}

1. Callon M, Lascoumes P, Barthe Y. Agir dans un monde incertain: essai sor la democratie technique. Paris: Seuil; 2001.

2. Avdeef A. The art of living: un mouvement indien au-delà des clivages religieux? Archives de Sciences Sociales des Religions. 2010;(149):169-187.

3. Altglas V. Le nouvel hindouisme occidental. Paris: CNRS Éditions; 2005.
4. Funes M. Notas sobre el concepto de estrés como clave de interpretación del mundo en el arte de vivir. Mitológicas. 2012;27:61-73.

5. D'Angelo A. Temblores y otras manifestaciones de la energía: la técnica del Sudarshan Kriya de El Arte de Vivir. Cultura y Religión. 2014;8(2):148-165.

6. Vargas $\mathrm{P}$, Viotti N. "Prosperidad y espiritualismo para todos": un análisis sobre la noción de emprendedor en eventos masivos de Buenos Aires. Horizontes Antropológicos. 2013;19(40):343-364. 
7. Viotti N. El affaire Ravi Shankar: neo-hinduismo y medios de comunicación en Argentina. Sociedad y Religión. 2015;25(43):13-46.

8. Viotti N, Funes ME. La política de la Nueva Era: el arte de vivir en Argentina. Debates do NER. 2015;2(28):17-36.

9. D'Angelo A. Al final todos terminaron viniendo como a terapia. El yoga entre la complementariedad pragmática, el trabajo terapéutico y la reorientación del self. Astrolabio Nueva Época. 2014;(12):193-225.

10. Seman P, Viotti N. "El paraíso está dentro de nosotros": la espiritualidad de la Nueva Era, ayer y hoy. Nueva Sociedad. 2015;(260):81-94.

11. Stolze Lima T, Goldman M. ¿Cómo se hace una gran división? Publicar en Antropología y Ciencias Sociales. 1998;6(7):155-167.

12. Latour B. Nunca fuimos modernos: ensayos de antropología simétrica. Buenos Aires: Siglo XXI Editores; 2007.

13. Law J. After method: mess in social science research. New York: Routledge; 2004.

14. Bellah R, Madsen R, Sullivan WM, Swidler A, Tipton SM. Habits of the heart: individualism and commitment in american life. Berkeley: University of California Press; 1985

15. Heelas $\mathrm{P}$, Woodhead L. The spiritual revolution: why religion is giving way to spirituality. Oxford: Wiley-Blackwell; 2005.

16. Champion F, Hervieu-Leger D, directores. De I'émotion en religión: renouveaux et traditions. Paris: Centurion; 1990.

17. Wood M. The sociology of spirituality: reflections on a problematic endeavor. In: Turner $B$, editor. The new Blackwell companion to the sociology of religion. London: Wiley-Blackwell; 2010. p. 267-285.

18. Wood M, Bunn C. Strategy in a religious network: a bourdieuian critique of the sociology of spirituality. Sociology. 2009;43(2):286-303.

19. Bender V, McRoberts O, Working Group on Spirituality Political Engagement and Public Life. Mapping a field: why and how to study spirituality [Internet]. New York: Social Science Research Council; 2012 [citado 25 jul 2017]. Disponible en: https://tinyurl.com/y7g7nufy.

20. Puglisi R. La "energía" que crea y sana: representaciones corporales y prácticas terapéuticas en devotos de Sai Baba. Ciencias Sociales y Religión/ Ciências Sociais e Religião. 2015;17(22):71-89.

21. Freidin B, Borda P. Identidades profesionales heterodoxas: el caso de médicas, médicos y psicólogas que integran medicinas y terapias alternativas en Argentina. Trabajo y Sociedad. 2015;(25):75-98

22. Bordes M. Esto de "alternativo" no tiene nada: la construcción de la razonabilidad del uso de una medicina no-convencional desde la perspectiva de sus usuarios en Buenos Aires, Argentina. Physis: Revista de Saúde Coletiva. 2015;25(4):1229-1249.

23. Saizar M. Reflexiones en torno de la complementariedad terapéutica entre usuarios del yoga en el Área Metropolitana de Buenos Aires (Argentina). Mitológicas. 2006;21:23-46.

24. Freidin $B$, Ballesteros $M$, Echeconea $M$. En búsqueda del equilibrio: salud, bienestar y vida cotidiana entre seguidores del ayurveda en Buenos Aires. En: Documentos de Trabajo No. 65 [Internet]. Buenos Aires: Instituto de Investigaciones Gino Germani; 2013 [citado 25 jul 2017]. Disponible en: https://tinyurl.com/yagtxazg.

25. Saizar M, Bordes M. Espiritualidad y otros motivos de elección de terapias alternativas en Buenos Aires. Mitológicas. 2014;29:9-24.

26. Carozzi MJ. Nueva era y terapias alternativas: construyendo significados en el discurso y la interacción. Buenos Aires: EDUCA; 2000.

27. Viotti N. El lugar de la creencia y la transformación religiosa en las clases medias de Buenos Aires. Apuntes de Investigación del CECyP. 2010;(18):39-68.

28. Toniol R. O que faz a espiritualidade? Religião e Sociedade. 2017;(37):144-175.

29. Giumbelli E, Toniol R. What is spirituality for? New relations between religion, health and public spaces. In: Mapril J, Blanes R, Giumbelli E, Wiolson E, editors. Secularisms in a Postsecular Age? Religiosities and Subjectivities in Comparative Perspective. New York: Palgrave; 2017.

30. Frigerio A. La “¿nueva?” espiritualidad: ontología, epistemología y sociología de un concepto controvertido. Ciencias Sociales y Religión/ Ciências Sociais e Religião. 2016;18(24):209-231.

31. Frigerio A. Lógicas y límites de la apropiación new age: donde se detiene el sincretismo. En: De la Torre R, Gutiérrez Zúñiga C, Juárez Huet N, editores. Variaciones y apropiaciones latinoamericanas del new age. México DF: CIESAS; 2013. p. 47-70. 
32. Gioberchio G. "La mente y la respiración tienen un rol muy importante para curar el cuerpo". Clarín [Internet] 6 sep 2012 [citado 10 ago 2017]. Disponible en: https://tinyurl.com/ yb4c2avd.

33. Blanco Villalba M. Todo tratamiento contra el cáncer debe tener un soporte científico. Clarín [Internet]. 6 sep 2012 [citado 10 ago 2017]. Disponible en: https://tinyurl.com/y8m7w3ud.
34. Callon M, Law J. After the individual in society: lessons on collectivity from science, technology and society. Canadian Journal of Sociology. 1997;22(2):165-182.

35. Mol A. The body multiple: ontology in medical practice. Durham: Duke University Press; 2003.

\section{FORMA DE CITAR}

Viotti N. Más allá de la terapia y la religión: una aproximación relacional a la construcción espiritual del bienestar. Salud Colectiva. 2018;14(2):241-256. doi: 10.18294/sc.2018.1519.

Recibido: 9 de julio de 2017 | Versión final: 3 de diciembre de 2017 | Aprobado: 17 de diciembre de 2017

Este obra está bajo una licencia de Creative Commons Reconocimiento-NoComercial 4.0 